\title{
Automated Global Feature Analyzer - A Driver for Tier-Scalable Reconnaissance
}

\author{
Wolfgang Fink ${ }^{(1)}$, Ankur Datta ${ }^{(2)}$, James M. Dohm ${ }^{(3,4)}$, Mark A. Tarbell ${ }^{(1)}$, Farrah M. Jobling ${ }^{(5)}$, Roberto Furfaro ${ }^{(6)}$, \\ Jeffrey S. Kargel ${ }^{(3)}$, Dirk Schulze-Makuch ${ }^{(7)}$, Victor R. Baker ${ }^{(3,4)}$ \\ ${ }^{(1)}$ California Institute of Technology, Visual and Autonomous Exploration Systems Research Laboratory, Division of \\ Physics, Mathematics and Astronomy, Mail Code 103-33, Pasadena, CA 91125, USA, \\ Email: wfink@autonomy.caltech.edu \\ ${ }^{(2)}$ Robotics Institute, Carnegie Mellon University, Pittsburgh, PA, USA \\ ${ }^{(3)}$ Department of Hydrology and Water Resources, University of Arizona, Tucson, AZ, USA \\ ${ }^{(4)}$ Lunar and Planetary Laboratory, University of Arizona, Tucson, AZ, USA \\ ${ }^{(5)}$ University of Colorado School of Medicine, Department of Microbiology, Aurora, CO, USA \\ ${ }^{(6)}$ Aerospace and Mechanical Engineering Department, University of Arizona, Tucson, AZ, USA \\ ${ }^{(7)}$ School of Earth and Environmental Sciences, Washington State University, Pullman, WA, USA
}

Abstract - For the purposes of space flight, reconnaissance field geologists have trained to become astronauts. However, the initial forays to Mars and other planetary bodies have been done by purely robotic craft. Therefore, training and equipping a robotic craft with the sensory and cognitive capabilities of a field geologist to form a science craft is a necessary prerequisite. Numerous steps are necessary in order for a science craft to be able to map, analyze, and characterize a geologic field site, as well as effectively formulate working hypotheses. We report on the continued development of the integrated software system AGFA: Automated Global Feature Analyzer ${ }^{\circ}$, originated by Fink at Caltech and his collaborators in 2001. AGFA is an automatic and feature-driven target characterization system that operates in an imaged operational area, such as a geologic field site on a remote planetary surface. AGFA performs automated target identification and detection through segmentation, providing for feature extraction, classification, and prioritization within mapped or imaged operational areas at different length scales and resolutions, depending on the vantage point (e.g., spaceborne, airborne, or ground). AGFA extracts features such as target size, color, albedo, vesicularity, and angularity. Based on the extracted features, AGFA summarizes the mapped operational area numerically and flags targets of "interest", i.e., targets that exhibit sufficient anomaly within the feature space. AGFA enables automated science analysis aboard robotic spacecraft, and, embedded in tier-scalable reconnaissance mission architectures, is a driver of future intelligent and autonomous robotic planetary exploration. ${ }^{12}$

\section{TABLE OF CONTENTS}

1. INTRODUCTION. 1

2. RATIONALE FOR AUtOMATED GEOLOGIC

Classification OF OPERATIONAL AREAS...............3

3. METHODS \& TECHNICAL IMPLEMENTATION .......3

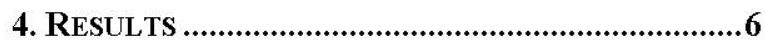

5. DISCUSSION \& OUTLOOK …………………………....8

1

${ }^{1} 1-4244-1488-1 / 08 / \$ 25.00$ @2 2008 IEEE.

${ }^{2}$ IEEEAC paper \#1273, Version 10, Updated December 14, 2007
REFERENCES............................................................... 9

BIOGRAPHY ................................................................ 10

\section{INTRODUCTION}

A multinational Mars exploration program is currently underway. Future missions involve multiple spacecraft, which are planned to orbit or land on Mars within the next decade. Varied instrumentation on these spacecraft have been generating huge datasets, and will generate unprecedented amounts of data in the future. In addition, as more and more missions spread out into the Solar System (e.g., Moon, Venus, Mercury, Titan, Pluto, various asteroids and comets), other potential problems arise, including a communications bottleneck as missions compete for downlink and uplink time, straining deep-space communications. This will result in extensive communication time lags, which translate into increased mission expenses. These and other potential problems using traditional onboard software make it infeasible to explore remotely large expanses of planetary surfaces independent from Earth control. Missions with traditional technologies will have to be replaced with autonomous "science craft" in order to make future missions more communication efficient, economical, and science effective, such as in situations where decisions, observations, and actions need to be made in-situ, e.g., to follow up on transient events. A traditional rover mission, for example, collects information at each stop along its planned traverse. This scenario is time and personnel intensive, and thus extremely costly. Just as significant, vital information may be bypassed along the traverse path. To construct a coherent history of what has transpired in the area of interest over geologic time, the reconnaissance field geologist moves from one patch of rock materials (or outcrops) to another over varying geological terrains. While tracking location and considering regional information previously compiled from published geological (e.g., stratigraphic, paleotectonic, paleoerosional, etc.), topographic, geophysical (e.g., gravity and magnetics), and hydrological (e.g., paleodischarge, drainage density, etc.) information, the field geologist gains a local 
and regional perspective of the geological, hydrological, environmental, and climatological histories of a chosen study site by gathering essential field data while en route. A comprehensive understanding of the geology, hydrology, environment, and climate by the field geologist can optimally be achieved by coupling regional information with high-resolution information carefully compiled while covering large expanses.

Traditional missions to planetary bodies such as Mars, Venus, and Titan have tended to focus either on exploring a single site with a rover or lander, or global mapping, such as with an orbiter. The former analyzes a site in depth at the expense of a regional understanding, while the latter returns immense datasets that are time and personnel intensive to compile and evaluate, but often overlook the local and regional geological significance.

Robotic reconnaissance operations are called for in extreme environments such as space (including planetary atmospheres, surfaces, and subsurfaces), and hazardous or inaccessible areas on Earth. A fundamentally new planetary exploration mission concept, Tier-Scalable Reconnaissance $^{\complement}$, originated by Fink et al. [1-6] (Fig. 1), replaces engineering and safety constrained mission designs that perform local ground-level reconnaissance with rovers and landers, or global mapping with an orbiter. The tierscalable paradigm integrates multi-tier (orbit $\Leftrightarrow$ atmosphere $\Leftrightarrow$ surface/subsurface) and multi-agent (orbiter(s) $\Leftrightarrow$ blimps $\Leftrightarrow$ surface/subsurface agents or sensors) hierarchical mission architectures, and enables reconnaissance in real time (e.g., monitoring of transient events) on global, regional, and local scales for high science return and fully autonomous robotic missions, providing independence from human intervention, yet permitting manual override at any level. Tier-scalable reconnaissance not only introduces mission redundancy and safety, but enables distributed, science-driven, and less constrained reconnaissance (both spatially and temporally) of prime locations on Mars, Moon, Titan, Venus, and elsewhere. Such tier-scalable reconnaissance missions require a high degree of operational autonomy, such as [5]:

1. Automatic mapping of an operational area from different vantage points (i.e., space, airborne, surface, subsurface);

2. Automatic feature extraction and target/region-ofinterest/anomaly identification within the mapped operational area, which includes intelligent data downlink;

3. Automatic target prioritization for follow-up or closeup (in-situ) examination;

4. Subsequent automatic (targeted) deployment and (concurrent) navigation/relocation of entire tiers or agents/sensors within tiers.
In place of astronaut geologists, the initial exploration of Mars and other planetary bodies will be conducted by robotic spacecraft. Therefore, training and equipping a robotic craft with the sensory and cognitive capabilities of a planetary/field geologist is a necessary prerequisite. Numerous steps are necessary in order for such a "science craft" to be able to automatically map, analyze, and characterize an operational area, and effectively formulate working hypotheses. Its main benefit will be the potentially increased science return during the respective mission lifetime and thus greater cost effectiveness.

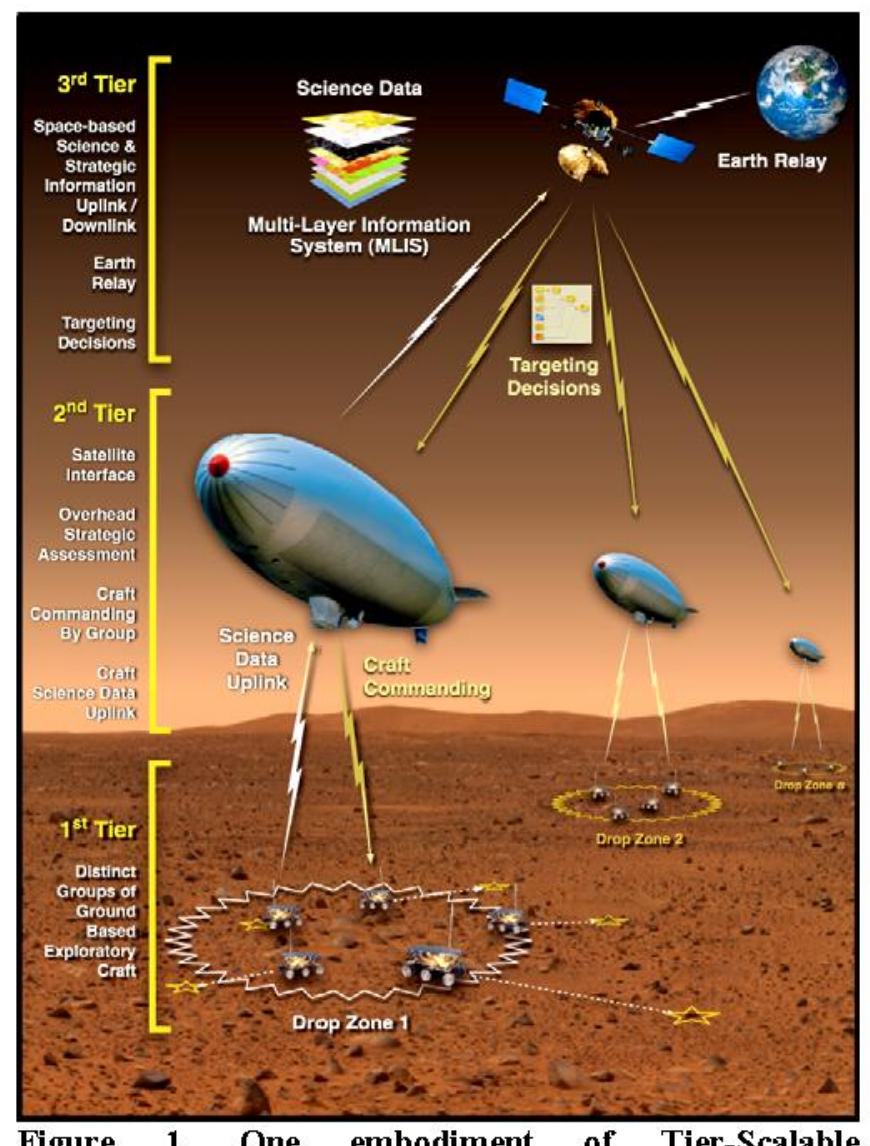

Figure 1. One embodiment of Tier-Scalable Reconnaissance $^{(}$: Tri-level hierarchical multi-agent architecture for autonomous remote planetary exploration (from [5], after [1]).

We report on the continued development of the integrated software system $A G F A^{\oplus}$ : Automated Global Feature Analyzer $^{(}$, originated by Fink at Caltech and his collaborators in 2001 (formerly Automated Geologic Field Analyzer $^{(0)}$ [7]). AGFA is an automatic and feature-driven target characterization system that operates in an imaged operational area, such as a geologic field site on a remote planetary surface. AGFA performs automated target identification/detection, feature extraction, feature classification, and target prioritization within mapped/imaged operational areas at different length scales and resolutions, depending on the vantage point (e.g., spaceborne, airborne, or ground). AGFA extracts features such as target size, color, albedo, vesicularity, and 
angularity. Based on the extracted features, AGFA summarizes the mapped operational area numerically and flags targets of "interest", i.e., targets that exhibit sufficient anomalous character within the feature space. AGFA enables automated science analysis for robotic spacecraft, and, embedded in tier-scalable reconnaissance mission architectures, is a driver of future intelligent and autonomous robotic planetary exploration.

\section{RATIONALe For AUtOMATED GeOLOGIC}

\section{Classification of Operational Areas}

In one embodiment of AGFA we focus in the following on the geologic classification of operational areas on a planetary surface, such as geologic field sites encountered by a planetary rover (or, to a much more limited degree, by a lander).

Why is it important to characterize the rocks, which compose the surface of a planet? The rocks reveal extensive information about the geological processes that transformed the surface. The shope of a rock can tell much about its location relative to its origin. Angular rocks tend to imply a short transport distance from their point of origin, while rounded ones tend to reflect a considerable distance involved from their place of origin. Alternatively, wellrounded rocks may also reflect the smoothing properties of various media, such as mud slurries or liquid water. The orientation of rocks may also reflect the presence of sustained liquid water. The size of rocks relative to other stones in their composite medium is another important indicator to the field geologist. If the rocks are of a common size, it may reflect a process, which sorted them as such. Very often on Earth, an outcrop, which displays the size of the rocks becoming finer as the field geologist moves up the stratigraphic column, is considered an important indicator of fluvial activity, including floods that deposited the materials. Alternatively, if the rocks are a mix of varying sized, shaped, and textured rock materials (e.g., boulders to grains of sand and/or muddy matrix), a catastrophic depositional process such as mass wasting might be a viable explanation for the emplacement of the materials. The color of rocks is also an important indicator of origin. Various lithologies such as basalt and sandstone have a characteristic range of colors they may display, depending on chemical composition and the environmental/weathering conditions during and after emplacement. The surface and surface texture of a rock also holds clues to its origin. If the rock is composed of other smaller rocks (also known as a composite rock and/or conglomerate), it may imply certain chemical bonding and/or depositional processes that can only occur in certain environments. If the rock displays fine layering, it may be sedimentary in nature, which also implies certain environments for its formation. Whether a rock is smooth or vesicular, combined with other rock attributes, may also be indicative of its origin. A highly vesicular, dark gray, sub-angular to angular rock, especially with phenocrysts, for example, would be best explained by a volcanic origin.

A synthesis of these important rock features listed above and others will in turn provide a greater understanding of rock types, process-driven activities, depositional environments, potential sources for the observed rock materials, and a coherent interpretation of the past geologic evolution.

It should be noted that during the reconnaissance of a geologic field site it is not so important to capture all the details, e.g., number of rocks and rock types, but to get a first good estimate and overview of what is there. Based on this first, "rough" estimate, a working hypothesis may be drafted, and based on this hypothesis, further corroborating or refined information may have to be gathered within the same or, in addition, within other, potentially distant operational areas. This "general procedure" - the geologic approach - led to the creation of AGFA.

\section{Methods \& TECHNICAL IMPLEMENTATION}

The aim of this paper is to undertake an important foundational step in developing and implementing an innovative and integrated software system, AGFA, for automated (science) analysis of operational areas. AGFA performs automated mapping/imaging, feature extraction, feature analysis, and target prioritization within operational areas at different length scales and resolutions, depending on the vantage point (e.g., spaceborne, airborne, or ground).

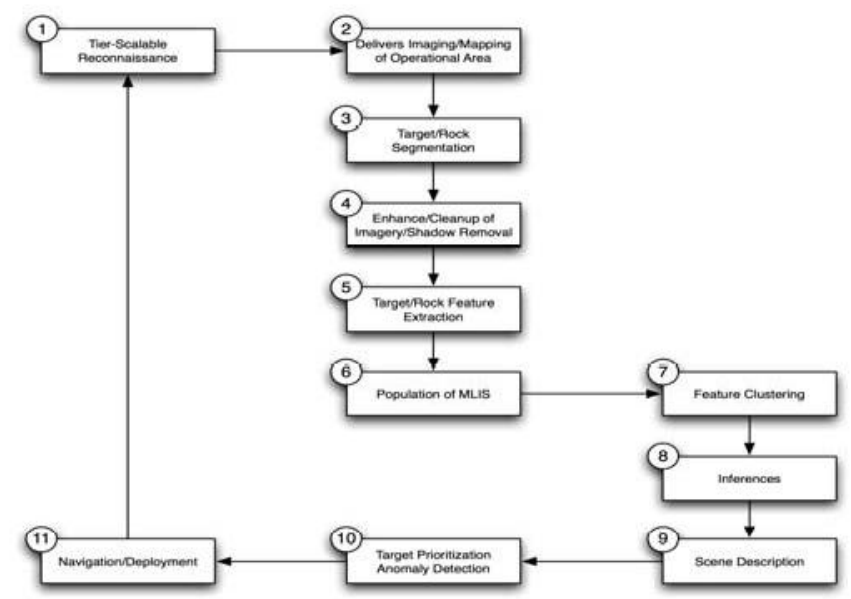

Figure 2. AGFA Operational Diagram (after [7]).

Employed by a planetary explorer such as an orbiter, balloon/blimp, or rover, AGFA (1) automatically maps and characterizes target/rock materials in the imaged operational area (e.g., geologic field site), both on the ground and from the air, including size (boulder, cobble, pebble), color, albedo (light, medium, dark), texture (vesicular or smooth), 
and shape (degree of angularity - angular, subrounded, rounded), and (2) identifies autonomously (i.e., exclusively feature-driven as opposed to "biased", human hypothesisdriven) "interesting" or anomalous rocks based on the extracted rock features. AGFA is an extensible analysis and classification framework, which is not limited to the currently implemented suite of methods that are discussed in the following (see also "Discussion \& Outlook"). We describe briefly the functional steps of AGFA and how they build on each other (Fig. 2; the detailed mathematical underpinning of AGFA will be provided elsewhere).

1. Imaging/Mapping - Images of the operational area under investigation are obtained at different length scales and resolutions (Fig. 3), depending on the vantage point (e.g., spaceborne, airborne, or ground).

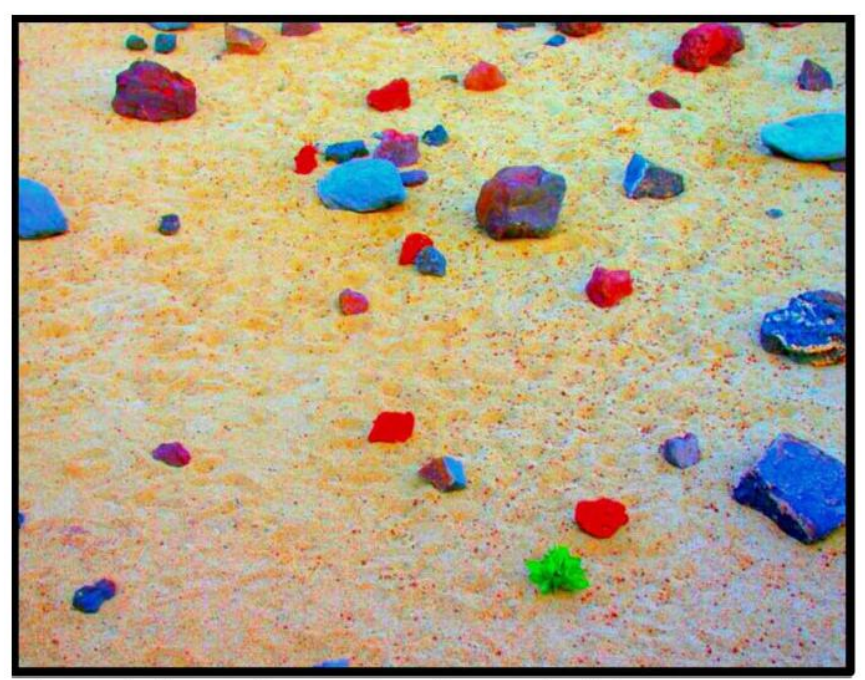

Figure 3. Imaged operational area, in this case a geologic field site as seen by a planetary rover (from [7]).

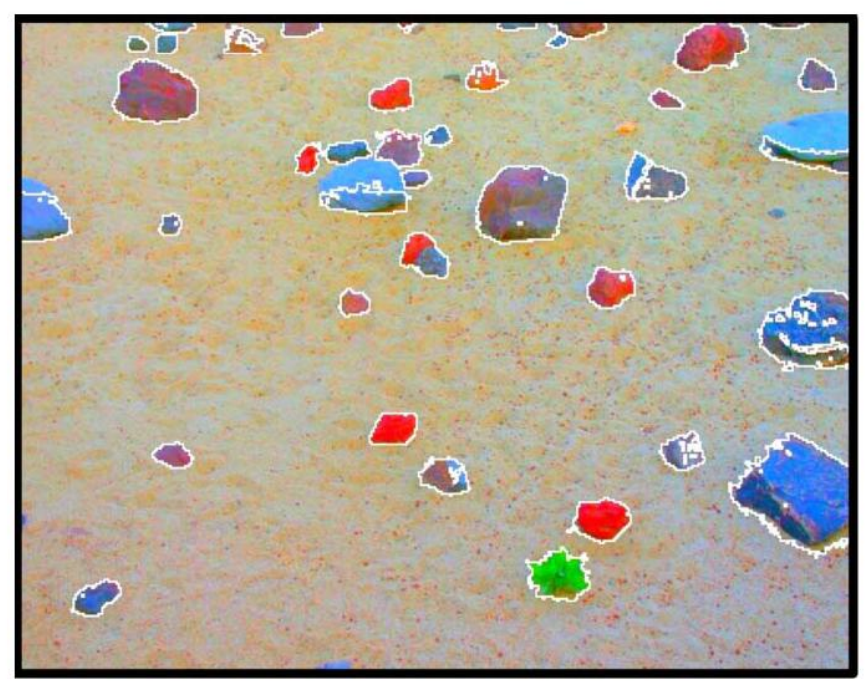

Figure 4. AGFA-performed image segmentation to identify targets (here: rocks) within the imaged operational area (from [7]).
2. Rock Segmentation - To segment out the rocks from the background of an imaged operational area we have developed and implemented several image segmentation schemes such as $k$-means and histogrambased segmentation for the purpose of clustering rocks and the background into separate clusters (Fig. 4).

3. Imagery Enhancement/Clean-up - Statistics based on the luminance and chrominance of the foreground surrounding a rock are derived and coupled with spatial constraints to remove pixels that have a high probability of being cast shadows

4. Target Feature Extraction - Targets/rocks that were identified in the previous steps are further characterized by extracting features such as size, color, albedo, texture, and shape descriptors. We have developed modules that currently give us the following feature measurements for each rock present in the imaged operational area:

- $\quad$ Size - The size of each rock is estimated using the image size (camera field of view) and the distance from the camera to the surface of the operational area. Rock sizes are currently subdivided into three classes: pebbles $(2 \mathrm{~cm}-10 \mathrm{~cm})$, cobbles $(10 \mathrm{~cm}-$ $20 \mathrm{~cm})$, and boulders $(>20 \mathrm{~cm})$. Rocks smaller than $2 \mathrm{~cm}$ are considered to be part of the background/matrix and are currently not characterized. The size and the following features would be equally extractable though when looking at microscopic images of operational areas.

- Color - The RGB color space is used in this module. The average RGB color of the rock is computed from its respective intensity distribution in each of the color channels.

- Albedo - The brightness or shade of an object provides significant information in its own right. The albedo is currently quantified into three ranges: light, medium, and dark. The perceived brightness of an object is a function of the incident light.

- Texture - Image texture is characterized by the spatial frequency and orientation of brightness variation in a region. Image texture can be used as an indicator of the three-dimensional surface texture of a rock. The resulting texture feature vector can be subdivided into classes indicating, for example, smooth, slightly to moderately vesicled, and highly vesicled rock surfaces. We have employed Gabor filters of different orientation and scale. 
- $2 D$ Shape Characterization - To characterize the shape of a target/rock we have developed and implemented the following modules:

a. Ellipse Fit - For each identified target/rock the best-fit ellipse is calculated to describe its shape, thereby obtaining measurements for the major axis a, the minor axis $b$, and the geometric eccentricity $e$, defined as the ratio of the semi-minor axis of the ellipse to the semimajor axis. The spatial orientation of the major/minor axes together with the absolute orientation of the operational area images allow for the calculation of an orientation vector field for targets/rocks in that region of interest, contributing to geologic process working hypotheses.

b. Angularity/Roundness - This measure indicates the sharpness of the corners of an object and the angularity of its edges. It is calculated as the deviation of the bestfit ellipse and the actual object outline. We distinguish between angular, subrounded, and rounded targets.

c. Eccentricity - The geometric eccentricity is defined as the ratio of the minor to the major axis of the best-fit ellipse for a given shape. It is a measure for the elongation of a particular object.

d. Extent-Extent is defined as the ratio of pixels that are inside the object, divided by the area of the bounding box around the object.

e. Moments - Moments are shape encoders and can be used to distinguish between different rock shapes. At present, we calculate $7 \mathrm{HU}$ and 10 Alt moments for each rock.

- Spectral Data - As a future extension to the AGFA software system we envision to incorporate a rock spectra software module that compares a particular observed rock spectrum to a database of stored, known rock spectra via artificial neural networks or stochastically optimized spectral retrieval procedures (e.g., [8]) to help determine the rock composition.

In applying steps 1 through 4, AGFA obtains feature vectors for all identified targets/rocks within the imaged operational area (Fig. 5). These feature vectors are subsequently used by AGFA to classify targets/rocks, and to identify anomalies. It is essential to note that this is done in an objective, i.e., exclusively feature-driven and thus unbiased automatic manner. In contrast to Artificial Intelligence (AI) schemes (e.g., $[9,10]$ ), AGFA does not depend on expert-defined rule sets, but operates instead within the classification-inherent feature space itself.

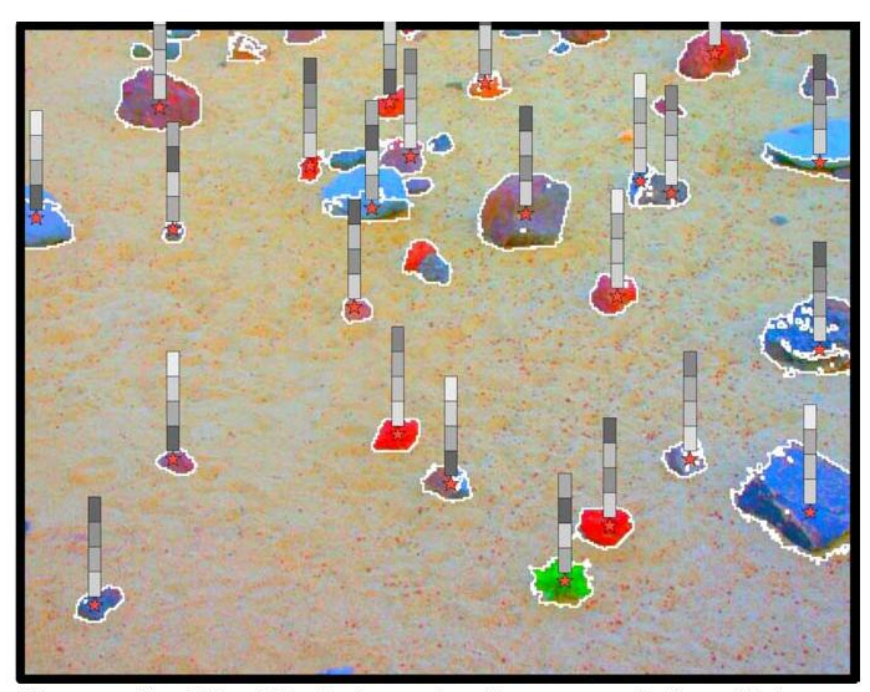

Figure 5. Identified targets (here: rocks) within an imaged operational area and their respective extracted feature vectors, derived by AGFA for the ground level. At this level, the targets can scale from microscopic soil particles to large rocks. For airborne and spaceborne vantage points, the targets scale from large rocks to mountain ranges (from [7]).

5. Feature Summary - The imaged operational area is described by first identifying regions of targets/rocks with common properties and subsequently by summarizing the values of the properties of the targets/rocks in these identified regions.

- Characterization of operational area: Having quantified the properties of individual rocks and identified regions of rocks, we summarize the properties of the imaged operational area. The percentage of area covered by rocks for each of the categories defined for all of the descriptive properties - size, color, albedo, texture, and shape can be calculated. In addition, the sorting of the rocks based on size can be evaluated (e.g., rocksize distribution)

6. Feature Clustering - The extracted, individual target feature vectors undergo a normalization process and are subsequently clustered into the natural number of clusters.

7. Target Prioritization - Multiple prioritization scenarios can be conceived to evaluate the importance of individual targets or combinations of targets to be further examined during reconnaissance missions, which differ in their respective level of complexity. 
These scenarios can range from simple feature-based or feature-clustering-based prioritization [11-13] to prioritization via context-based clustering [14].

Recently, more advanced prioritization frameworks [15] have been developed using previously acquired, coarse feature/reconnaissance data that was preclustered using either general purpose clustering algorithms [11-13]) or clustering algorithms associated with special-purpose models [14]. This advanced class of algorithms facilitates (1) the selection of single or multiple targets, and (2) the selection of instruments used for the close-up examination of these targets in an operational area for potential information gain about the operational area under investigation [15].

8. Inference \& Anomaly Detection - Based on the clusters obtained from the previous steps, two flags are calculated, the distonce flag and the number flag:

- Distance Flag - The distance flag compares the distance between the cluster centers to their respective size in the feature space: if the sum of the respective largest eigenvalue for each cluster, obtained via Principal Component Analysis (PCA), is less than the distance between the centers of the respective two clusters, then the distance flag is set to red, else green.

- Number Flag - The number flag tries to estimate how abnormal the population size of a cluster (i.e., number of cluster members) is compared to another: If there is a cluster, which is only a fraction of the size of the cluster it is compared to, then the number flag is set to red, else green.

The most interesting cases arise from a red-red flag combination, indicating absolute anomalies. Relative anomalies are indicated in cases of red-green or greenred flag combinations. The least interesting situations are given by a green-green flag combination, in which case no anomalies are indicated. More refined classification schemes, other than just three decision cases, can be applied (see also $[9,10]$ for real-valued decision functions).

Based on AGFA's feature summary, inference \& anomaly detection, and resulting target prioritization, subsequent automatic (targeted) deployment and navigation/relocation of entire tiers or agents/sensors within tiers of a tier-scalable reconnaissance mission architecture can occur (see also "Discussion \& Outlook"). As such, AGFA becomes the actual driver for tier-scalable reconnaissance missions.

We have developed an overall architecture for AGFA in both MATLAB and C, wherein the user can interactively choose the features to be calculated. AGFA can also operate in batch-mode, i.e., automatically generating feature vectors according to a pre-specified list of features without any further user intervention.

\section{Results}

In the following we present two example classifications performed by AGFA in its geologic embodiment: (1) an artificial scene (Fig. 6) to demonstrate in simple terms how AGFA operates, and (2) a "real-world" application of AGFA (Fig. 7).

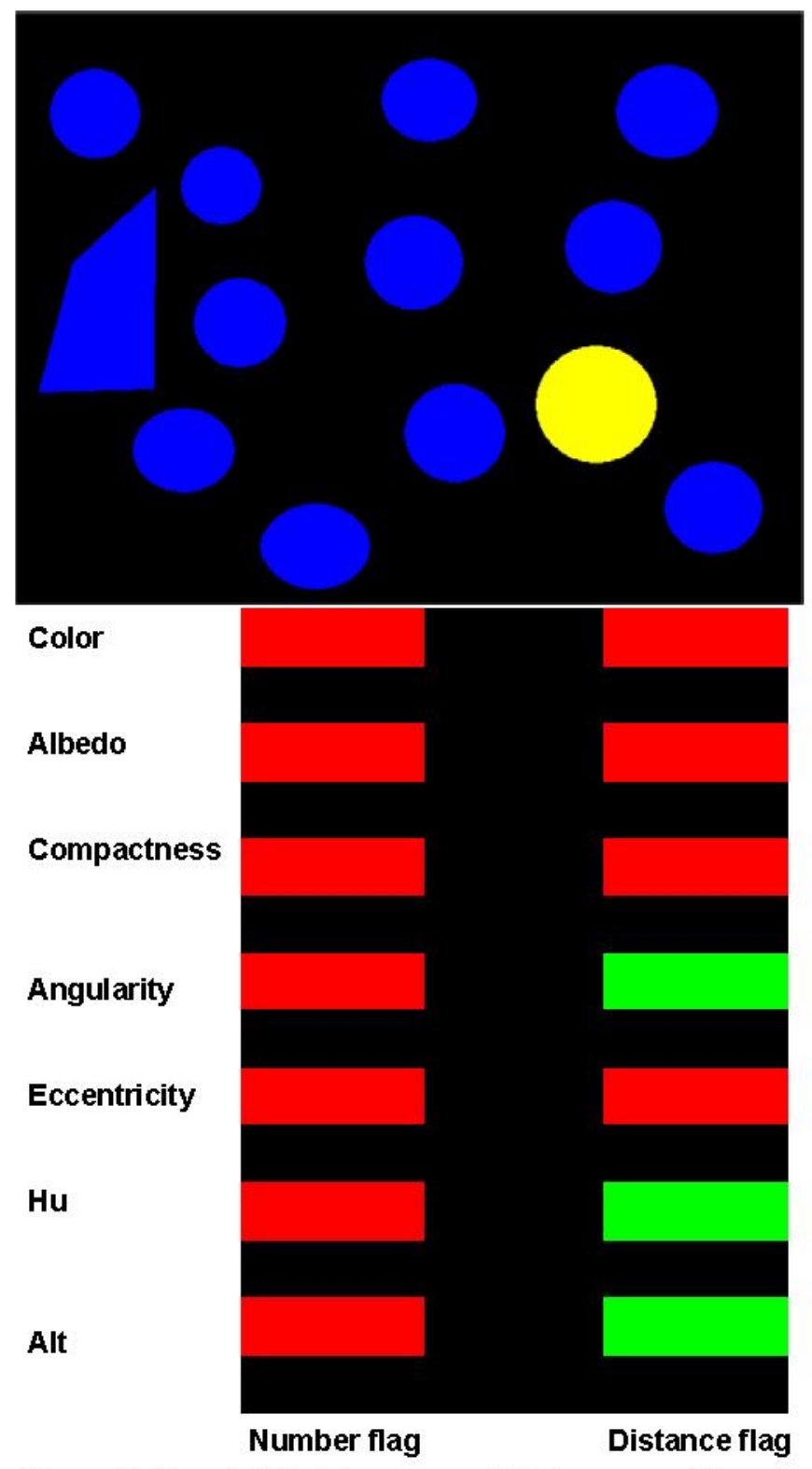

Figure 6. Top: Artificial scene, containing several targets that differ in shape, color, and number. Bottom: AGFAclassification result, indicating (1) absolute anomalies in the color, albedo, compactness, and eccentricity feature spaces, and (2) relative anomalies in the angularity and in the shape-encoders $\mathrm{Hu}$ and Alt. 


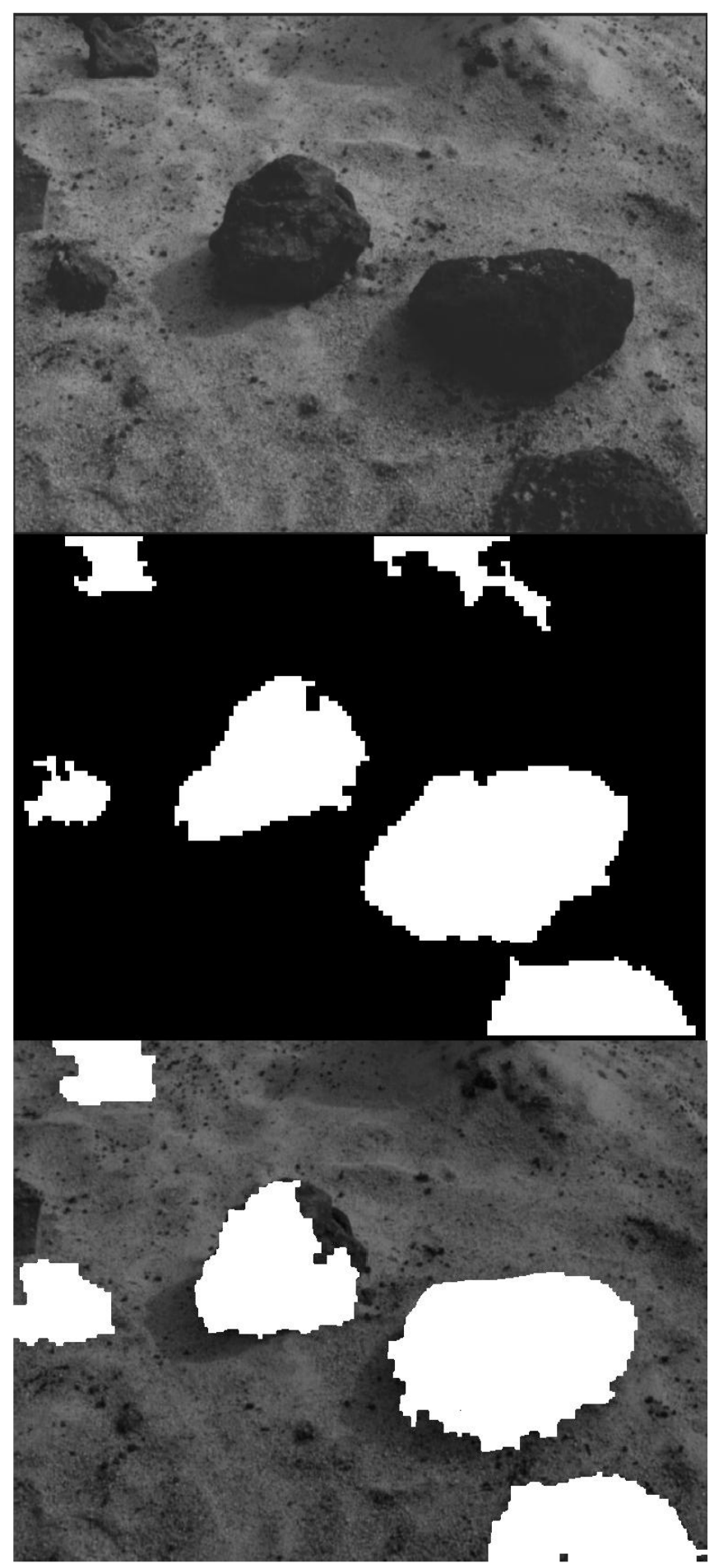

Figure 7. The six picture panels depict an example analysis sequence of AGFA, performed on an image of a more realistic scene. $P 1$ : original image of operational area; $P 2$ : identified/segmented target areas (rocks); $P 3$ : shadow removal in identified rock areas; $P 4$ : ellipse fitting of identified rock areas together with orientation of semi-major axes; $P 5$ : numerical feature summary of identified targets; and P6: resulting AGFA-classification of entire imaged operational area: in this case, no anomalies detected.

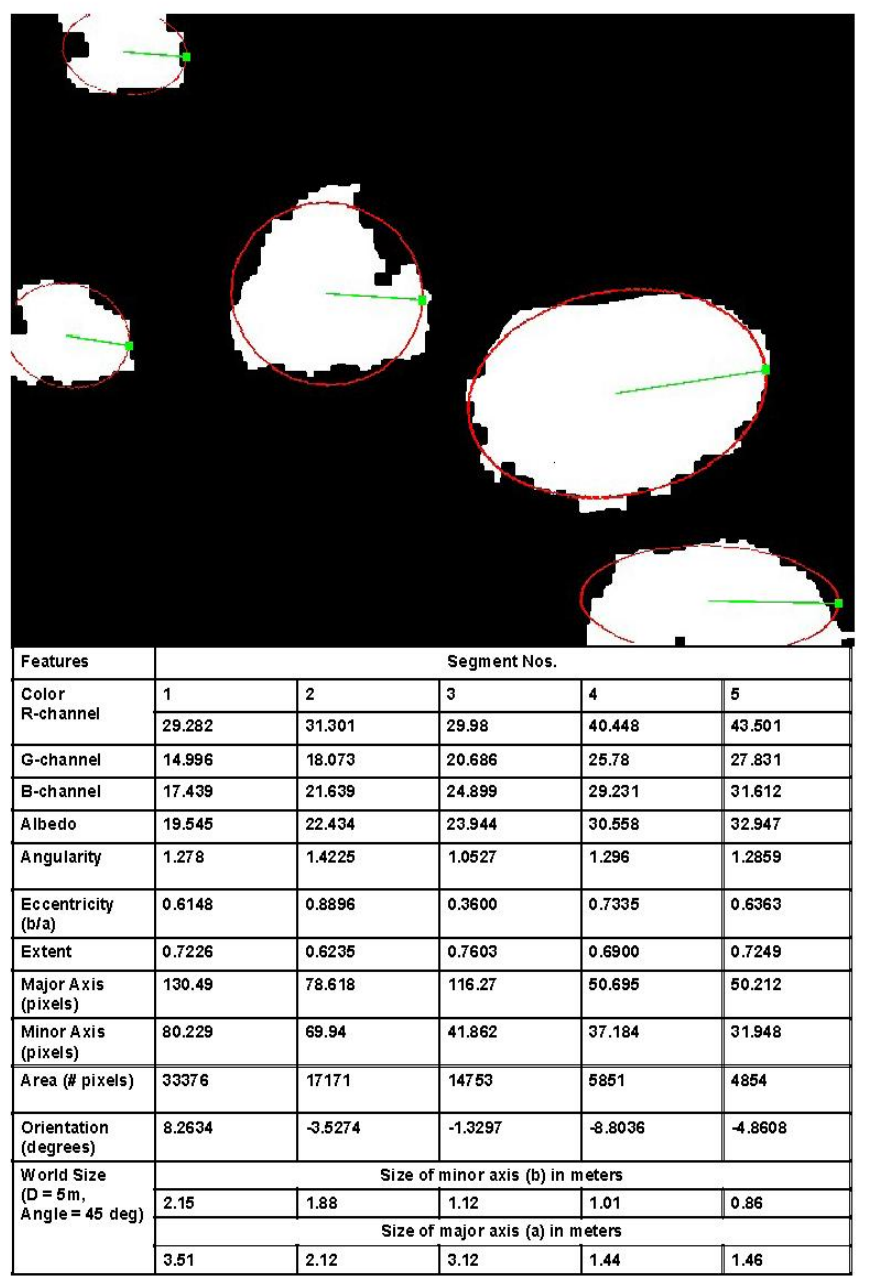

Color

Albedo

Compactness

Angularity

Eccentricity

$\mathrm{Hu}$

Alt

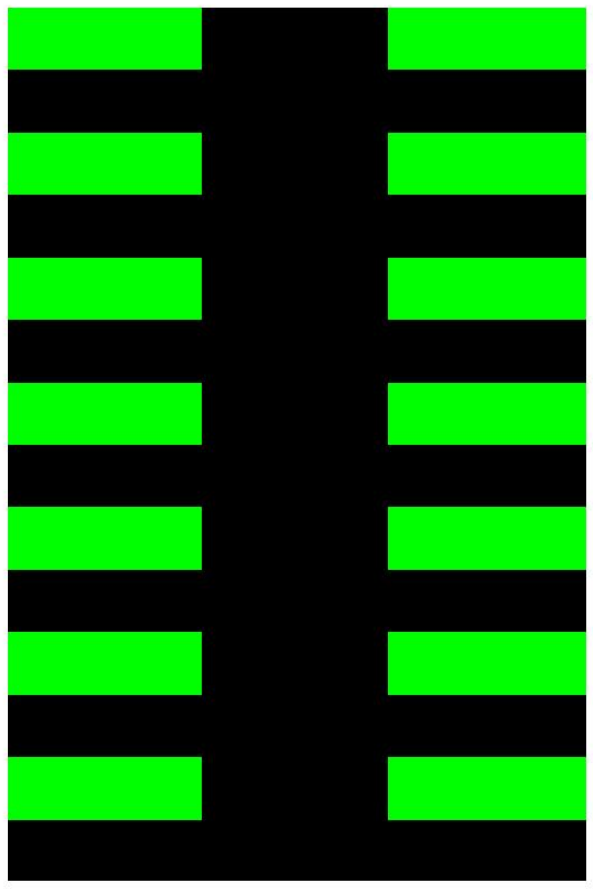

Number flag 


\section{Discussion \& OUTLOOK}

There have been several elements of autonomous/automated science analysis packages previously proposed and developed such as in [16]. These elements have generally been applicable only under specialized conditions and must be applied manually. An autonomous system for a characterization of an operational area (here: geologic field site) that incorporates a variety of elements to develop a comprehensive understanding of a region has not been demonstrated to the best of our knowledge.

In contrast, AGFA is a first-of-a-kind approach towards a fully automated (no human-in-the-loop) and integrated software system that performs image segmentation of an operational area, such as a geologic field site on Mars, Moon, Titan, or Earth, thereby acquiring (science) targets, followed by a comprehensive feature extraction (e.g., shape, size, color, albedo, texture, angularity, eccentricity, compactness, extent) for the identified targets, which is then followed by an objective and self-contained (i.e., exclusively feature-driven as opposed to "biased", human hypothesis-driven) anomaly detection, employing a variety of clustering algorithms such as sequential and hierarchical clustering algorithms. The AGFA software system enables a fully automated and comprehensive characterization of an operational area such as a geologic field site on a remote planetary surface.

An additional strength of AGFA is the fact that, when embedded into a tier-scalable reconnaissance architecture, it enables, for the first time, the intelligent exploration of remote planetary surfaces not only from the ground but from the air and space as well. For example, aerial reconnaissance from a balloon, blimp, airship, or glider on Mars, Titan, or Venus in addition to or in place of groundbased rover units, could cover a tremendously larger area of the surface in a much shorter time than previously possible $[1-6,17,18]$.

The study of geology is fundamentally different from the other sciences, such as physics or chemistry. Whereas these fields rely on fundamental laws derived from mathematics and experimentation, geology has always been the art of inferring the natural history based on the signs in the present. Geology has discovered many unique and amazing phenomena, from plate tectonics (continental drift) to the former existence of gigantic glacial ice sheets. But none of these processes was discovered by direct observation. None was determined from fundamental laws. All the advancements of understanding the natural world that geology has achieved have come from abduction or retroduction [19], with subsequent deduction. The deduction is usually never made from a single piece of evidence, rather, it is a suite of different and unique evidence, and often is difficult to discern in the field. It is for these reasons that we propose any comprehensive and accurate study of planetary surfaces (e.g., Mars) must be conducted in the manner that field geology has always operated. Given the cost and difficulty of sending human geologists into space, the next best thing would be an autonomous robotic science craft or several science craft as part of a tier-scalable mission architecture, equipped with the sensory capability and the ability to "reason" like a field geologist.

The reasons for this necessity are not always obvious. How does one characterize an area in terms of its geologic history? Examining the rocks, though having the potential to yield significant information, may not be enough to confidently formulate working hypotheses of the processesrealted activities and environmental, hydrological, and climatological conditions that contributed to the surface expression observed by the science craft. First, the science craft must be aware of the context it is in. To a field geologist, the implications of the materials around him can mean very different things if he is in a canyon as opposed to a mountaintop. Next, he must be aware of the relationships between the materials around him. Can they be grouped into categories of similar origin or substance? Can he identify different levels or terraces that may differentiate some materials from others? How do different materials relate to each other? Does one type of material (called a unit or layer) lie on top or below another? Or are the units tilted, folded, or show evidence of inversion? What are the types of landforms that occur in the region of study, and how do these landforms occur in time and space? All this information must be obtained, but much more importantly, analyzed as to the relationships between each other and the area they occupy. This is absolutely critical. This kind of analysis was achieved with the Pathfinder rover mission, but only after the information was sent back to Earth and analyzed by teams of human geologists. Considering the size and incredible differences in terrain and material composing the surface of Mars [20-25], this method would not allow for a thorough understanding of the planet in a manageable time span. Instead of this time and energy consuming process, the advantage of an autonomous robotic field geologist becomes apparent.

Our goal is to eventually merge AGFA with other innovative tools for autonomous science analysis of geological field sites, including GIS-based multi-layer information, which includes published geological, topographic, geophysical, mineralogic, and hydrological data sets at local, regional, and global scales. This MultiLayer Information System (MLIS) [1-3] (Fig. 8), containing geologic, structural, and erosional information, combined with AGFA capabilities could help unravel complex geologic histories at local, regional, and global scales that can be readily updated with information obtained from future tier-scalable reconnaissance missions (Fig. 1).

Although the focus of this paper is mainly on geologic classification of operational areas, future embodiments of 
AGFA will address (1) geophysical, (2) geochemical, (3) (hyper-)spectral, and (4) biological classifications of operational areas for enhanced geologic and exobiologic exploration.

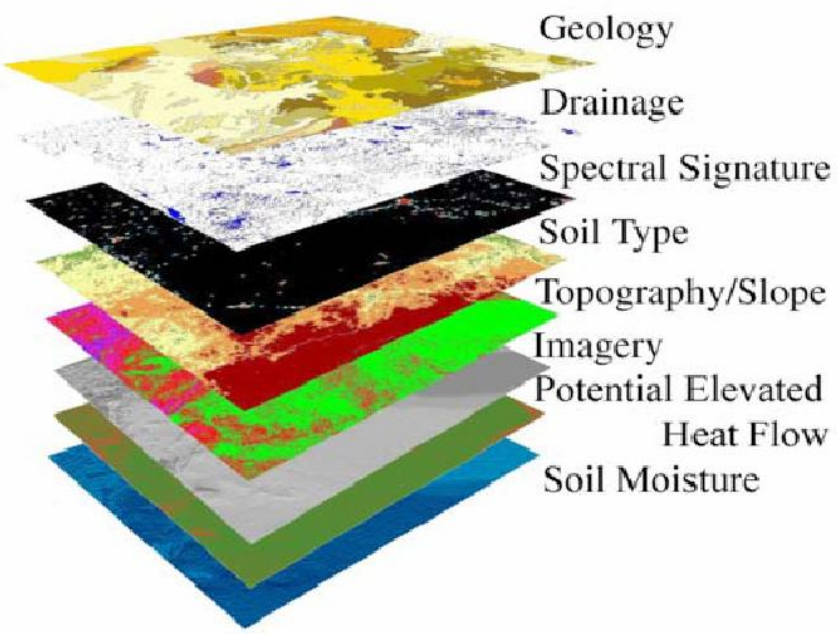

Figure 8. Example layers, in part delivered by AGFA, which make up the Multi-Layer Information System (MLIS) (from [1-3]).

AGFA is currently undergoing field testing aboard groundbased robotic platforms (Fig. 9) $[4,5]$ and will be tested in the near future aboard airborne platforms, such as helicopters and blimps, as part of the tier-scalable reconnaissance mission test bed at Caltech [4]. As for the spaceborne perspective, AGFA will be tested on satellite and orbiter imagery, and for a close-up (microscopic) ground perspective, it will be tested on microscopic images, such as those delivered by the Mars Exploration Rovers (MER) Spirit and Opportunity (e.g., [26]).

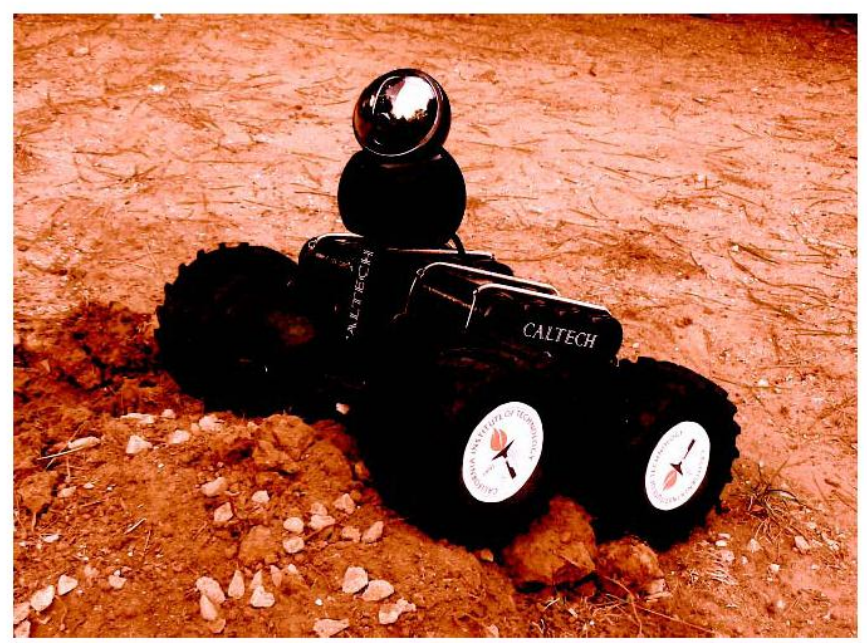

Figure 9. 4WD remote controllable robotic platform as a representative mobile ground-tier agent of the tierscalable reconnaissance mission test bed at Caltech (from $[4,5]$ ). This platform is being used for testing AGFA in an operational environment.

\section{REFERENCES}

[1] Fink W, Dohm JM, Tarbell MA, Hare TM, Baker VR (2005) Next-Generation Robotic Planetary Reconnaissance Missions: A Paradigm Shift; Planetary and Space Science, 53, 1419-1426.

[2] Fink W, Dohm JM, Tarbell MA, Hare TM, Baker VR, Schulze-Makuch D, Furfaro R, Fairén AG, Ferré TPA, Miyamoto H, Komatsu G, Mahaney WC (2006) Autonomous Tier-Scalable Reconnaissance Missions For Remote Planetary Exploration; Proceedings of the $4^{\text {th }}$ International Planetary Probe Workshop 2006, Pasadena.

[3] Fink W, Dohm JM, Tarbell MA, Hare TM, Baker VR, Schulze-Makuch D, Furfaro R, Fairen AG, Ferre TPA, Miyamoto H, Komatsu G, Mahaney WC (2007) TierScalable Reconnaissance Missions For The Autonomous Exploration Of Planetary Bodies; IEEE Aerospace Conference Proceedings, Big Sky, Montana.

[4] Fink W and Tarbell MA (2007) Tier-scalable Reconnaissance Mission Test Bed: Implementation of Ground-Tier [abstract 2410]; $38^{\text {th }}$ Lunar and Planetary Science Conference Abstracts [CD-ROM], Lunar and Planetary Institute, Houston.

[5] Fink W, George T, Tarbell MA (2007) Tier-Scalable Reconnaissance: The Challenge of Sensor Optimization, Sensor Deployment, Sensor Fusion, and Sensor Interoperability; SPIE Defense \& Security Symposium Proceedings, Orlando, Florida.

[6] Fink W. Caltech's Visual and Autonomous Exploration Systems Research Laboratory. News Media Releases on Tier-Scalable Reconnaissance. [http://autonomy.caltech.edu/autonomy/tierscalable.html].

[7] Fink W, Datta A, Baker V (2005) AGFA: (Airborne) Automated Geologic Field Analyzer; Geochimica et Cosmochimica Acta, Volume 69, Number 10S, A535.

[8] Terrile RJ, Fink W, Huntsberger TL, Lee S, Tisdale ER, Tinetti G, von Allmen P (2005) Retrieval of Extra-Solar Planetary Spectra Using Evolutionary Computation Methods; Division for Planetary Sciences (DPS) 37th Meeting of the American Astronomical Society, Cambridge, UK, Bull. Amer. Astron. Soc., 37, 31.19.

[9] Furfaro R, Dohm JM, Fink W (2006) Fuzzy Logic Expert System for Tier-scalable Planetary Reconnaissance; $9^{\text {th }}$ International Conference on Space Operations, AIAA, Rome, Italy, June 19-23, 2006. 
[10] Furfaro R, Dohm JM, Fink W, Kargel JS, SchulzeMakuch D, Fairén AG, Ferré PT, Palmero-Rodriguez A, Baker VR, Hare TM, Tarbell M, Miyamoto HH, Komatsu G (2007) The Search for Life Beyond Earth Through Fuzzy Expert Systems; Plonetary and Space Science (in press).

[11] Duda RO, et al. (2000) Pattern Classification and Scene Analysis, John Wiley \& Sons, $2^{\text {nd }}$ edition.

[12] Bishop CM (1995) Neural Networks for Pattern Recognition, Clarendon Press, Oxford.

[13] Williams CKI (2000) An MCMC Approach to Hierarchical Mixture Modelling, Advances in Neural Information Processing Systems 12, S. A. Solla, T. K. Leen, K.-R. Mueller, eds., MIT Press.

[14] Fink W, et al. (2001) Clustering Algorithm for Mutually Constraining Heterogeneous Features Technical Report JPL-ICTR-01-5.

[15] Fink W (2006) Generic Prioritization Framework for Target Selection and Instrument Usage for Reconnaissance Mission Autonomy, Proceedings of IEEE World Congress on Computational Intelligence (WCCI) 2006, Vancouver, Canada, 11116-11119.

[16] Gulick VC, Morris RL, Ruzon MA, Roush TL (2001) "Autonomous image analyses during the 1999 Marsokhod rover field test", Journal of Geophysical Research, Vol. 106, No. E4, 7745-7763.

[17] Schulze-Makuch D, Dohm JM, Fairén AG, Baker VR, Fink W, Strom RG (2005) Venus, Mars, and the Ices on Mercury and the Moon: Astrobiological Implications and Proposed Mission Designs; Astrobiology, 5, 778-795.

[18] Noor AK, Cutts JA, Balint TS (2007) Platforms for discovery: Exploring Titan and Venus; Aerospace America/June 2007.

[19] Baker, V.R, 1999, Geosemiosis; Geological Saciety of America Bulletin, v. 111, p. 633-646.

[20] Dohm, J. M., and K. L. Tanaka (1999) Geology of the Thaumasia region, Mars: plateau development, valley origins, and magmatic evolution, Plonetary and Space Science, 47, 411-431.

[21] Dohm, J. M., V. R. Baker, R. C. Anderson, J. C. Ferris, T. M. Hare, K. L. Tanaka, J. E. Klemaszewski, D. H. Scott, and J. A. Skinner (2000) Martian magmatic-driven hydrothermal Sites: potential sources of energy, water, and life, presented in the Concepts and Approaches for Mars exploration: Lunor and Plonetary Inst., Houston, LPI Contrib. No. 1062, 93-94.
[22] Dohm, J. M., R. C. Anderson, V. R. Baker, J. C. Ferris, L. P. Rudd, T. M. Hare, J. W. Jr. Rice, R.R. Casavant, R. G. Strom, J.R, Zimbelman, and D. H. Scott (2001) Latent activity for western Tharsis, Mars: significant flood record exposed, J. Geophys. Res., 106, 12,301-12,314.

[23] Dohm, J.M., K. L. Tanaka, and T. Hare (2001) Geologic map of the Thaumasia region of Mars: USGS Misc. Inv. Ser. Mop I-2650, scale 1:5,000,000.

[24] Dohm, J.M., Ferris, J.C., Baker, V.R, Anderson, R.C., Hare, T.M., Strom, R.G., Barlow, N.G., Tanaka, K.L., Klemaszewski, J.E., and Scott, D.H. (2001) Ancient drainage basin of the Tharsis region, Mars: Potential source for outflow channel systems and putative oceans or paleolakes, J. Geophys. Res.

[25] Schulze-Makuch, Dohm JM, Fan C, Fairen AG, Rodriguez JAP, Baker VR, Fink W (2007) Exploration of Hydrothermal Targets on Mars; Icanus, doi:10.1016/j.icarus.2007.02.007.

[26] Cabrol NA, Grin EA, Herkenhoff K, Richter L, Athena Science Team (2007) Soil Sedimentology, Textures and Dynamics at Gusev Crater from Spirit's Microscopic Imager [abstract 1784]; $38^{\text {th }}$ Lunar and Planetary Science Conference Abstracts [CD-ROM], Lunar and Planetary Institute, Houston.

\section{BIOGRAPHY}

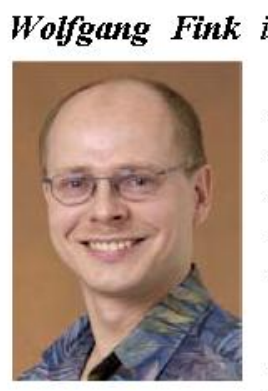

Senior Researcher at NASA's Jet Propulsion Laboratory in Pasadena, CA, Research Associate Professor of both Ophthalmology and Ne urological Surgery at the University of Southern California, Los Angeles, CA, and Visiting Associate in Physics at the California Institute of Technology, Pasadena, $\mathrm{CA}$. $\mathrm{He}$ is the founder and head of the Visual and Autonomous Exploration Systems Research Laboratory at Caltech (http://autonomy.caltechedu). His research interests include autonomous planetary and space exploration, computational field geology, computer optimization, image processing and analysis, sensor data fusion, astrobiology, and biomedicine. Dr. Fink obtained a B.S. and MS. degree in Physics and Physical Chemistry from the University of Göttingen and $a$ Ph.D. in Theoretical Physics from the University of Tübingen in 1997. His work is documented in numerous publications and patents. Dr. Fink holds a Commercial Pilots License for Rotorcraft. 
Ankur Datta is currently a PhD student at the Robotics

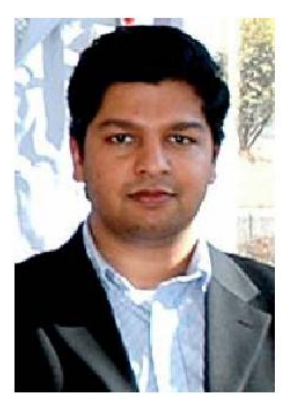
Institute in the School of Computer Science at Carnegie Mellon University. He obtained a bachelors degree from the University of Central Florida in 2004. His research interest include building autonomous computer vision algorithms to make robots perceptive. He has participated in several NSF Research Experience for Undergraduates (REU) programs and his work is documented in publications at ICPR, IEEE ICME and IASTED Graphics and Image Processing conferences. He was selected to participate in a summer program organized by JPL and Caltech in 2003. In 2004, he was the CRA (Computer Research Association) Outstanding Undergraduate Award Finalist and also won the prestigious NSF Graduate Fellowship.

James M. Dohm is a Planetary Geologist and Senior

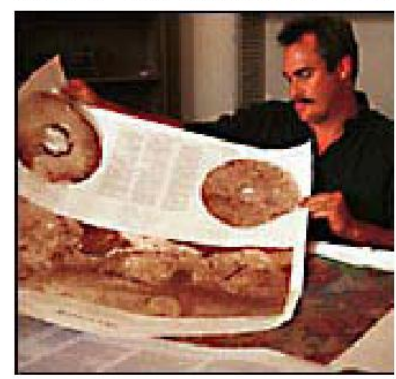
Researcher at the University of Arizona. He performs planetary investigations at local to global scales. He has extensive geological field experience coupled with more than 19 years of experience with planetary geological research, which includes 12 years as assistant coordinator of the NASA Mars and Venus Programs (MGM and VGM, respectively), now known as the Planetary Mapping Program. In addition, James Dohm has been recently involved with satellite and rover missions as a science team member of the Life in the Atacama Rover Field Experiment, Sensor Web, the "Subsurface Access" project of the Mars Technology Program, the Autonomous Sciencecraft Experiment, and the Nomad Rover Experiment. He has contributed to the publication of 6 USGS I maps at four map scales, more than 60 peer-reviewed journal articles, and more than five book publications.

Mark A. Tarbell is a Senior Software Specialist with more

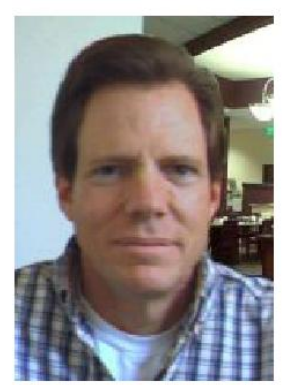
than 15 years of satellite and groundbased command and control system architecture design and development. Tarbell designed and implemented the ground data processor control infrastructure for JPL's recent SRTM mission, and was involved with JPL's Jason JTCCS project, which supports real-time telecommanding of Earthorbiting satellites from wireless handheld PDAs. In collaboration with the Visual and Autonomous Exploration Systems Research Laboratory at Caltech, he recently co-designed and implemented a remote telecommanding control system for an indoor test bed for autonomous surface exploration at Caltech's Visual and Autonomous Exploration Systems Research Laboratory.

Farrah M. Jobling is currently a Research Associate at the

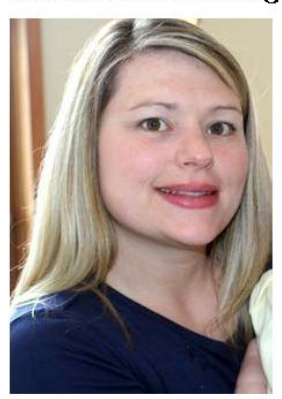
University of Colorado, School of Medicine, Dept. of Microbiology. Dr. Jobling obtained a BA in Biochemistry from Whittier College and a Ph.D in Molecular Microbiology and Immunology from the University of Southern California. Her research has expanded on her interest in heavy metal toxicity and gene regulation in response to exposure to metals. Currently, her research involves bacterial pathogenesis and gene regulation in response to essential elements, such as iron, in iron limiting environments. Her other main interest is her adorable new son.

Roberto Furfaro is currently Assistant Research Professor

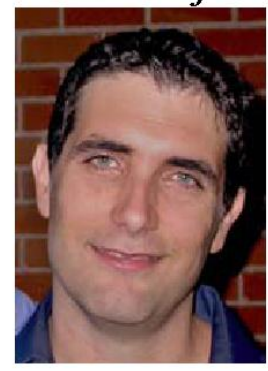
in the Aerospace and Mechanical Engineering Department, University of Arizona. He has a large spectrum of research interests, which includes neutron and photon computational transport, neural and fuzzy systems, space systems and micro-satellite design. Over the past few years, he has been collaborating with Ecosystem Science and Technology branch at NASA Ames on the "NASA Coffee Project" in which he led the development of an intelligent algorithm for coffee ripeness prediction using UAV airborne images. He has had a long-term involvement with Mars exploration since 1998 when he joined the NASA SERC at University of Arizona to become the project manager for the development of two robotic devices designed to utilize Martian local resources. Recently, he has been working developing of novel engineering solutions for planetary exploration including fuzzy-based expert systems for autonomous life-searching in extraterrestrial bodies.

Jeffrey $\boldsymbol{S}$. Kargel, an adjunct professor at the University of

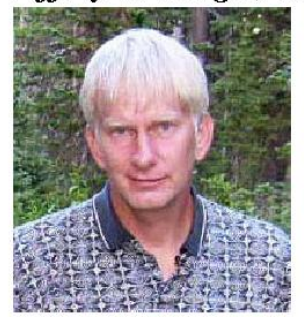
Arizona, is a geologist (B.S., M.S., Geological Sciences, Ohio State University) and planetary scientist (PhD, 1990, Planetary Sciences, University of Arizona) and an expert in cryospheric systems (glaciers and permafrost). He is the Principle Investigator of Global Land Ice Measurements from Space (GLIMS) and leads or participates in a variety of planetary research projects. He has authored or coauthored over 70 peer-reviewed papers, including several on the topic of asteroid and Mars resources. 
Dirk Schulge-Makuch is Associate Professor at

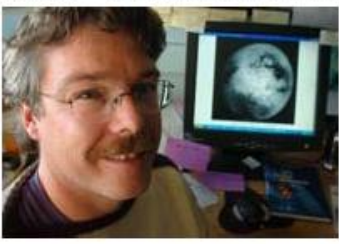

Washington State University. His most recent scientific accomplishments are the publication of his book "Life in the Universe: Expectations and Constraints" (Springer Publ., Berlin, 2004) along with many refereed papers in international journals. His research is centered on a broad range of topics with astrobiological relevance.

Victor A. Baker is Regents' Professor of the University of

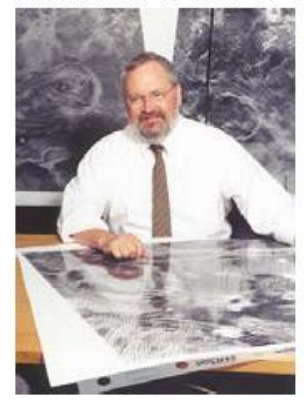
Arizona in the departments of hydrology and water resources, planetary sciences and geosciences. He has more than 30 years experience in planetary science research, particularly in geological studies of Mors and Venus. He also has had long experience with interpretive studies of terrestrial remote sensing, especially in regard to his specialties in fluvial geomorphology and flood hydrology. Dr. Baker is a Fellow of the American Geophysical Union, Honorary Fellow of the European Geosciences Union, Fellow of the American Association for the Advancement of Science, and Foreign Member of the Polish Academy of Sciences. He was the 1998 President of The Geological Society of America, and he holds the 2001 Distinguished Scientist Award from the Quaternary Geology and Geomorphology Division of that society. He is author or editor of 14 scholarly books or monogrophs, more than 300 scientific papers and chapters, and over 400 published abstracts and short reports. 